\title{
Antibiotic dispensing practices during COVID-19 and implications for Antimicrobial Resistance (AMR): Parallel mystery client studies in Uganda and Tanzania
}

Emmanuel Olamijuwon ( $\nabla$ emmanuel@olamijuwon.com )

University of St Andrews

Eveline Konje

Catholic University of Health and Allied Sciences

Catherine Kansiime

Mbarara University of Science and Technology

Mike Kesby

University of St Andrews

Katherine Keenan

University of St Andrews

Stella Neema

Makerere University

Benon Asiimwe

Makerere University

Stephen E. Mshana

Catholic University of Health and Allied Sciences

Martha F. Mushi

Catholic University of Health and Allied Sciences

Olga Loza

University of St Andrews

Benjamin Sunday

Mbarara University of Science and Technology

Alison Sandeman

University of St Andrews

Derek J. Sloan

University of St Andrews

Fernando Benitez-Paez

University of St Andrews

Joseph R. Mwanga

National Institute for Medical Research 


\section{Wilber Sabiiti}

University of St Andrews

Matthew T. G. Holden

University of St Andrews

Joel Bazira

Mbarara University of Science and Technology

Christine Muhumuza

Makerere University

Ivan Muhwezi

Mbarara University of Science and Technology

Kathryn Jean Fredricks

University of St Andrews

\section{Research Article}

Keywords: COVID-19, Uganda, Tanzania, drug sale, pharmacy, antimicrobial resistance, mystery client

Posted Date: January 24th, 2022

DOI: https://doi.org/10.21203/rs.3.rs-1276230/v1

License: (c) (1) This work is licensed under a Creative Commons Attribution 4.0 International License. Read Full License 


\section{Abstract}

Background: Over-the-counter antibiotic access is common in low-and-middle-income countries, including Tanzania and Uganda, and this may accelerate antimicrobial resistance. Our study explores critical aspects of the drug seller-client interaction and antibiotic dispensing patterns for simulated COVID-19 symptoms during the pandemic in two study sites in countries with different government responses to the pandemic.

Methods: Research assistants posing as clients approached different types of drug sellers such as pharmacies (Pharms), drug shops (DSs), and accredited drug dispensing outlets (ADDOs) in Mwanza, Tanzania ( $n$ Pharms $=415, n A D D O s=116$ ) and Mbarara, Uganda ( $n$ Pharms $=440, n D S s=67$ ), from June 10-July 30,2021 . The mystery clients (who held no prescription) sought advice for simulated COVID-19 symptoms from the drug sellers, documented the quality of their interaction with sellers and the type of drugs dispensed.

Results: Adherence to COVID-19 preventive measures and vigilance to COVID-19 symptoms was low in both sites but significantly higher in Uganda than in Tanzania. A higher percentage of drug sellers in Mbarara (Pharms $=36 \%$, DSs $=35 \%, p$-value $=0.947$ ) compared to Mwanza (Pharms $=9 \%$, ADDOs $=4 \%, p$ value $=0.112$ ) identified the client's symptoms as possibly COVID-19. More than three-quarters of drug sellers in both Mbarara (Pharms= 86\%, DSs $=89 \%$ ) and Mwanza (Pharms $=93 \%$, ADDOs= 97\%) sold prescription-only medicines without asking for a prescription. A relatively high percentage of drug sellers that sold prescription-only medicines in Mwanza (Pharms= 51\%, ADDOs=67\%) compared to Mbarara (Pharms $=31 \%$, DSs $=42 \%$ ) sold a partial course without any objection. Among the drug sellers who sold antibiotics, more drug sellers in Mbarara (Pharms $=73 \%$, DSs $=78 \%, p$-value $=0.580$ ) compared to Mwanza (Pharms $=40 \%$ ADDOs $=46 \%, p$-value $=0.537$ ) sold antibiotics relevant for treating secondary bacterial infections in COVID-19 patients.

Conclusion: Our study highlights low levels of awareness of and vigilance towards COVID-19 symptoms and widespread propensity to dispense prescription-only antibiotics without a prescription and to dispense partial doses of antibiotics. The implications of these for antimicrobial resistance are further discussed. Our study also highlights the need for more efforts to improve antibiotic stewardship among drug sellers in response to the current pandemic and to prepare them for future health emergencies.

\section{Introduction}

The direct impacts of COVID-19, in terms of morbidity and mortality, have been less severe in Africa compared with other world regions, influenced by a number of demographic and health systems factors, such as younger age structures, African governments' responses, and health system preparedness for infectious disease [1,2]. However, COVID-19 will likely have many serious indirect impacts on health and society in Africa. An area of particular concern is how COVID-19 response and preventative measures, and their unintended consequences, will affect the pre-existing and ongoing 'silent pandemic' of 
antimicrobial resistance (AMR). AMR, like COVID-19, is a global health threat that requires urgent attention [3]. Evidence suggests that the burden of AMR in low and middle-income countries (LMICs) is high compared to high-income countries [4]. There is a pressing need to understand the social factors that facilitate the biological development of AMR [5]. Among multiple social drivers, the motivations and behaviours of drug sellers and pharmacists play a critical role in AMR because they impact how people access antibiotics in LMICs, and because inappropriate dispensing practices may fuel drug resistance [6-8]. This paper investigates drug sellers' practices during the COVID-19 pandemic in sites in two countries (Mbarara, Uganda and Mwanza, Tanzania) with varying societal responses to the pandemic.

How the COVID-19 and AMR pandemics are interacting is unclear and likely to be context-dependent. A significant potential risk in LMICs is that COVID-19 prevention policies may drive an increase in antibiotic self-medication through over-the-counter purchases and a breakdown in antibiotic stewardship [9].

Several factors may be at play. Patients may avoid healthcare facilities when seeking treatment for other ailments, for fear of catching COVID-19 in these settings, or may be reluctant to present with COVID-19 like symptoms [10-12]. Interruptions in public healthcare provision for illnesses including HIV, malaria, and tuberculosis may have increased footfall at pharmacies and drug shops, which have remained open during lockdowns as essential services [13]. Finally, the COVID-19 'infodemic' [14], particularly its aspect which promotes misleading information about antibiotic use to prophylatically 'treat' COVID-19 [15,16] and which circulates in both mass and social media, promoted by a range of authorities from traditional and religious leaders to politicians on the world stage, may also have increased demand for the over-thecounter sale of antibiotics [17,18]. Alternatively, an increased focus on public health, risk avoidance and public health safety regulations may have improved antibiotic stewardship and dispensing practices.

In both Tanzania and Uganda, drug sellers operate under well-established regulatory frameworks. In Tanzania, they are comprised of two main types: type 1 pharmacies, which are supervised by a registered pharmacist and are permitted to sell a wide range of prescription-only medications including antibiotics; and type 2 (known officially as accredited drugs dispensing outlets - ADDOs - and colloquially as 'maduka ya dawa muhimu', literally, 'essential medicines shops'), which a suitably qualified[1] person can run after completing the five-week ADDO training course, but which can dispense a more limited range of drugs. ADDOs were established in 2003 as part of a national policy to address shortages in fully-qualified pharmacists and increase access to pharmaceutical drugs, including antimicrobials [19]. In 2019 there were 1,504 type 1 registered pharmacies and 14,045 ADDOs in Tanzania [20]. Chalker et al. [21] found that respondents favoured the latter over public health facilities as their preferred means to access antimicrobials [21]. Uganda, meanwhile, has two main types of drug sellers: Type 1 pharmacies, which are supervised by a qualified pharmacist and permitted to sell a wide range of prescription-only medications; and drug shops, which are managed by individuals with at least two years of health training or a health-related qualification and which can sell a limited range of medicines [22]. The small retail drug shops generally have minimal health infrastructure and often sell pharmaceutical drugs alongside other general services [22]. They are often the main source of pharmaceutical drugs in rural areas [23,24]. As in Tanzania, private drug shops in Uganda are well-regulated and inspected before approval [25]. 
Despite clear regulations stating that a prescription is required before antibiotics are dispensed, over-thecounter sales are common in many LMICs [22,26-28]. Mbonye et al. [29] showed that the majority of drug shops in Uganda sold antibiotics, especially amoxicillin and trimethoprim-sulfamethoxazole, even though they were not authorised to do so [30]. Drug sellers in Uganda were aware of national regulations and rarely displayed prohibited drugs on the shelf for fear of inspectors and penalties, but would provide the client with the same on demand [22]. Mystery client studies conducted as part of our consortium's earlier work on AMR recorded widespread non-compliance with regulations prohibiting antibiotic sale without a prescription in Tanzania [27,31]. The study found that most providers across three study regions dispensed amoxicillin on-demand without question, to clients with no prescription [27].

Notwithstanding this evidence of non-compliance with national regulations, there is little evidence on how antibiotic dispensing and sales have been affected by the COVID-19 pandemic. This study builds on an earlier, pre-COVID-19, project on the drivers of AMR in East Africa [32] to explore pandemic-era drug seller behaviours and practices in Tanzania and Uganda. The study was conducted in mid-2021, when healthcare facilities were struggling to cope with COVID-19 alongside the usual burden of disease, mitigation measures were still evolving, and both governments and citizens were attempting to adjust to the unprecedented conditions. This study investigates the following questions: To what extent do drug sellers in the different settings follow internationally recommended best practices for COVID-19 infection control related to hygiene, mask use and social distancing? How vigilant are drug sellers to COVID-19 like symptoms and what is the nature and quality of advice they give to clients presenting with such symptoms? Do drug sellers recommend antibiotics to clients reporting COVID-19 like symptoms?

Comparing drug dispensing practices in Uganda and Tanzania is important because although their antibiotic provision landscapes were similar pre-COVID-19, their early COVID-19 policy response diverged considerably in ways that may have shaped individual risk assessment, compliance with safety guidelines, and susceptibility to AMR [33]. During the pandemic, Uganda implemented stringent household lockdowns and social distancing regulations, including restrictions on public travel and closure of non-essential shops and markets. Tanzania's approach was less consistent: fluctuating from strict public health measures being in place (March-April 2020), through a period of so-called 'COVID denial' (April 2020 - early 2021) in which public health measures and testing capacity were rolled back [34,35]. Before 2021, Tanzania was one of the few countries that imposed few physical distancing measures $[33,36]$. Although residents were advised against physical contacts, such as shaking hands, there have been no major lockdowns or closures of non-essential activities in order to protect the country's economy, and no restrictions on public gatherings for religious activities [33,36]. Misinformation has also been prevalent in Tanzania. For example, spiritual remedies through prayers were promoted as strategies for combatting the pandemic [33,35]. With new leadership (March 2021 - present) the position is of stricter measures to curb the spread of the virus, such as the enforcement of mass masking mandates and the establishment of new testing centres.

[1] Suitably qualified persons are those with a preexisting relevant qualification in nursing, pharmacy or clinical training - or other qualification approved by the Ministry of Health. To qualify for training, the 
potential dispenser must be one of the following: Pharmaceutical technician, Pharmaceutical assistant, Nursing officer, Nurse midwife, Clinical officer, Clinical assistant, Nursing assistant, or any other person seen as capable by the MoHSW after being advised by Tanzania Food and Drug Authority.

\section{Methods}

\section{Study Design}

This cross-sectional community-based quantitative survey used a mystery client design, also referred to as a simulated client study, to assess drug-dispensing practices. Mystery client studies are commonly used to observe drug provider behaviour while minimising observation bias [21,27]. In our study, 27 male and female field workers, unknown to the sellers, posing as clients (hereafter known as mystery clients, or MCs) visited the pharmacies, ADDOs and drug shops. MCs were trained to follow the scenario strictly without improvising. MCs presented with COVID-19 like symptoms, reporting fever, cough, sore throat, loss of sense of taste and smell. MCs were provided with suggestions on how to respond during the encounter (full details in the online supplementary file). If asked further about symptoms, the MC replied that this was the first time they had experienced them (developed in the last 48 hours), that they had not visited a doctor, nor did they have a prescription, nor were they taking any other drugs. If offered any kind of test (for COVID-19 or other), they declined. If asked about their knowledge of COVID-19, they reported some knowledge but general confusion about symptoms. If the seller failed to recommend a pharmaceutical drug, the MC asked why they could not have amoxicillin (the drug they 'usually took for such symptoms') or even 'something stronger' since they were 'experiencing a lot of pain' (a statement designed to prompt sellers to challenge clients about the use and utility of antibiotics for the treatment of respiratory symptoms/pain and about the need for a prescription). If offered any pharmaceutical drug, the MC made one attempt to purchase 'only a couple of days' worth to see if it worked'. This response was designed to elicit sellers' advice that a full course of antibiotics should be purchased and consumed. After the encounter, MCs retired to a private space to record their interactions with the drug sellers on mobile devices installed with Epicollect5 [37], before moving to the next seller. To minimise the potential spread of the virus during fieldwork, the MCs were advised to keep a safe distance and use a hand sanitiser before visiting the next drug seller.

\section{Data collection and sample}

The sample frame built upon earlier work undertaken as part of a 3-country, interdisciplinary study of drivers of AMR in East Africa (Holistic Approach to Unravel Antibacterial Resistance in East Africa or HATUA), details published elsewhere [32]. Mwanza district in Tanzania and Mbarara district in Uganda were two of nine sites investigated by HATUA. Between April and July 2019, before the COVID-19 pandemic, we comprehensively mapped drug outlets within Mwanza and Mbarara districts to create a sample frame for mystery client studies $[27,31]$. Trained field assistants systematically traversed roads and pedestrian areas, using Global Positioning System (GPS) enabled tablets and phones and the 
software Epicollect5 [37] to record location and basic descriptive details about formal and informal outlets that sold medicine for humans [32]. At that time, 427 drug shops and 271 pharmacies were recorded in Mbarara in Uganda, while 504 ADDOs and 182 pharmacies were recorded in Mwanza, Tanzania. This initial sample frame was used to draw a convenience sample of 531 pharmaceutical drug sellers in Mwanza and 507 in Mbarara which were resurveyed between 10 June and 30 July 2021. The final sample analysed for each country comprised 116 pharmacies and 415 ADDOs in Mwanza, Tanzania, and 67 pharmacies and 440 drug shops in Mbarara, Uganda. Figure 1 presents the spatial distribution of drug sellers included in this study.

\section{Study variables}

During data collection, we recorded general information about the drug sellers surveyed, including the seller type, type of ownership, affiliation, size and perceived orderliness. All types of outlets are required to be clean and to have their licence and qualifications displayed $[25,38]$. The MCs observed the environment of the drug outlets and recorded whether a licence was visible in the shop, whether handwashing facilities, hand sanitiser and COVID-19 health information were available and visible around the shop, and whether intentional or accidental social distancing was observed.

MCs also recorded data on a range of issues. First, regarding vigilance: whether sellers suggested explicitly that the symptoms described might be COVID-19 and/or demonstrated any non-verbal signs (shock or alarm) that they were alert to this possibility. Second, regarding drug sellers' response: whether sellers asked if the symptoms were new or experienced previously, if a doctor had been consulted, and if the $\mathrm{MC}$ had a prescription or was taking other medications. Third, regarding advice provided: whether specific COVID-19 related advice was given during the encounter, including advice to get tested for COVID19 , self-isolate, maintain social distancing and/or wear a face mask as a safety precaution to protect others. Fourth, regarding dispensing practice: whether an antibiotic was sold, whether the MC or the seller had first suggested this, and whether the seller responded positively to the MC's request for only a couple of days' worth of medicine. If the seller agreed to sell 'a few days' worth', we considered this as 'sold a partial course' (see supplementary material Table $\mathbf{S 1}$ for a detailed description of the average number of tablets sold as 'a few days' worth/ partial course). Situations where sellers granted the client their wish for 'a few days' worth but advised them that they should buy and finish a full course were classified as 'sold partial course hesitantly'. The drug names and the number of pills dispensed were recorded in all cases. Finally, MCs recorded whether the drug seller gave clear instructions that a full minimum course should be taken even if they did not sell one on this occasion.

Data on the drugs sold were categorised in three ways: first, as either 'prescription-only' or 'over-thecounter' (if national regulations meant they could be sold without a prescription). Second, using the World Health Organisation (WHO) AWaRe classification of 'access', 'watch', and 'reserve' [39]. Third, we identified which drugs had been named in national guidelines as suitable for treating patients with confirmed, severe COVID-19 to control secondary bacterial co-infections. 


\section{Patient and public involvement}

We worked closely with government, research agencies and community stakeholders during the design, and data collection phase of the study. Preliminary findings have also been shared during a joint a stakeholders engagement meeting, a process which in turn fed into the analysis and interpretation of the data.

\section{Statistical analysis}

All responses from the fieldwork were retrieved from Epicollect5 and imported into R Studio for further cleaning, analysis, and visualisation. Frequency and percentage distributions were used to describe characteristics of drug sellers, the quality of interaction between MCs and drug sellers, measures of compliance with COVID-19 safety precautions and other patterns that emerged from the data. All analyses were stratified by drug seller type to delineate differences in drug dispensing practices between ADDOs and pharmacies. We used the chi-square test or Fisher exact test (in the case of small cell counts) to assess significant differences in drug dispensing practices between drug shops/ADDOs and pharmacies in both countries.

\section{Results}

\section{Characteristics of the drug outlets and COVID-19 preventative measures}

Table 1 presents a summary of the characteristics of the drug outlets surveyed. In both study sites, most pharmacy outlets sampled were considered 'large' or 'wholesale' pharmacies, while most drug shops or ADDOs were characterised as 'small' shops. In both countries, MCs subjectively assessed the general organisation and cleanliness of the shops as adequate in over $85 \%$ of cases; however, this was much higher among pharmacies than ADDOs/drug shops. In both study sites, pharmacies were more likely to display registration licences or certificates than drug shops or ADDOs. More than half of drug shops $(51 \%)$ and pharmacies $(70 \%)$ surveyed in Mbarara, Uganda had hand sanitiser available, compared with less than one-tenth of ADDOs (5\%) and pharmacies (7\%) surveyed in Mwanza, Tanzania. More than half of all drug seller types in both settings, however, have a handwashing facility. COVID-19 information was visible in $48 \%$ of Ugandan outlets, compared with only $7 \%$ of Tanzanian ones.

Table 1: Descriptive Characteristics of the Drug Sellers in Tanzania and Uganda 
Characteristics

Mbarara, Uganda

$\mathrm{N}$ (column \%)

Pharmacy
Drug shops
Mwanza, Tanzania

$\mathrm{N}$ (column \%)

All Pharmacy ADDO

Sellers
All

Sellers

\section{Size of the Facility}

Large/wholesale pharmacy

$57(85.1) \quad 13(3.0)$

70

(13.8)

Small shop

$10(14.9)$

427
$(97)$

437

(86.2)

$84(72.4)$

\begin{tabular}{ll}
6 & 90 \\
$(1.4)$ & $(16.9)$ \\
\hline 409 & 441 \\
$(98.6)$ & $(83.1)$
\end{tabular}

\section{General Organization}

Adequate - Clean | Orderly

Poor - Not clean | Not well

ordered

66 (98.5)

375

(85.2)

441
$(87)$

115

(99.1)

$333 \quad 448$

$1(1.5)$

65
$(14.8)$

$66(13) \quad 1(0.9)$

(80.2) (84.4)

License and certification

License/Certificate not Visible

$\begin{array}{llllll}13(19.4) & 133 & 146 & 9(7.8) & \begin{array}{l}164 \\ (39.5)\end{array} & \begin{array}{l}173 \\ (32.6)\end{array} \\ & (30.2) & (28.8) & & & \\ 54(80.6) & 307 & 361 & 107 & 251 & 358 \\ & (69.8) & (71.2) & (92.2) & (60.5) & (67.4)\end{array}$

\section{Presence of Hand Sanitizer}

Hand Sanitizer not Present

Hand Sanitizer Present

$\begin{array}{llllll}20(29.9) & 218 & 238 & 108 & 396 & 504 \\ & (49.5) & (46.9) & (93.1) & (95.4) & (94.9) \\ 47(70.1) & 222 & 269 & 8(6.9) & 19 & 27 \\ & (50.5) & (53.1) & & (4.6) & (5.1)\end{array}$

\section{Presence of Hand Washing}

Facility

Handwashing Facility not

Present

Handwashing Facility Present
$13(19.4) \quad 34(7.7)$

$54(80.6)$
406

(92.3)
47

(9.3)

460

(90.7) $\begin{array}{ll}82 & 83 \\ (19.8) & (15.6)\end{array}$

(4.6)

\section{Presence of COVID-19 Health Information}

\begin{tabular}{lllllll}
$\begin{array}{l}\text { COVID-19 Health Information } \\
\text { not Present }\end{array}$ & $34(50.7)$ & $\begin{array}{l}231 \\
(52.5)\end{array}$ & $\begin{array}{l}265 \\
(52.3)\end{array}$ & $\begin{array}{l}101 \\
(87.1)\end{array}$ & $\begin{array}{l}395 \\
(95.2)\end{array}$ & $\begin{array}{l}496 \\
(93.4)\end{array}$ \\
$\begin{array}{l}\text { CoVID-19 Health Information } \\
\text { Present }\end{array}$ & $33(49.3)$ & $\begin{array}{l}209 \\
(47.5)\end{array}$ & $\begin{array}{l}242 \\
(47.7)\end{array}$ & $15(12.9)$ & $\begin{array}{l}20 \\
(4.8)\end{array}$ & $\begin{array}{l}35 \\
(6.6)\end{array}$ \\
\hline Total & 67 & 440 & 507 & 116 & 415 & 531
\end{tabular}




\section{Vigilance to COVID-19 symptoms and implementation of international best practices on transmission avoidance}

The summary of seller's vigilance to COVID-19 symptoms and adherence to international guidance for preventing COVID-19 is presented in Figure 2. On all measures, drug sellers in Mbarara, Uganda were more alert to the potential threat of COVID-19 and were more likely to advise COVID-19 prevention measures than their counterparts in Mwanza, Tanzania. Only about $9 \%$ of pharmacies and $4 \%$ of ADDOs in Mwanza, Tanzania explicitly suggested that MCs might have COVID-19, compared to more than a third of the pharmacies and drug shops in Mbarara, Uganda (36\% and 35\% respectively). More sellers in Mbarara, Uganda were also 'surprised, shocked or alarmed' at the mention of COVID-19 symptoms than those in Mwanza, Tanzania.

While there were small differences in the advice given by different outlet types within the sites, crossnational differences were more striking. A significantly higher percentage of drug sellers in Mbarara, Uganda compared to Mwanza, Tanzania gave MCs some recommendations for limiting the spread of COVID-19, such as testing, wearing a face mask, social distancing, and self-isolation. In Mwanza,

Tanzania, drug sellers rarely implemented any common COVID-19 prevention practices, such as advising MCs to get tested, self-isolate, socially distance or wear a face mask. Less than $1 \%$ of drug sellers in ADDOs advised MCs to get tested, self-isolate, observe social distancing, or wear a face mask, compared to about $5 \%$ (or less) of pharmacies in the same study site. In Mbarara, Uganda, nearly a third of all drug sellers gave precautionary advice for limiting the spread of COVID-19. Among drug shops, $28 \%$ advised MCs to get tested, $8 \%$ to self-isolate, $26 \%$ to observe social distancing and $33 \%$ to wear a face mask.

\section{Dispensing practices and quality of drug seller-client interaction}

Figure 3 describes the attempts made to establish 'patient history' and the dispensing practices observed when sellers were presented with the study scenario across outlets, study sites and for different types of drug sellers. In Mbarara, Uganda, $41 \%$ of drug sellers asked MCs if they had experienced the symptoms before, compared to $32 \%$ in Mwanza, Tanzania. A minority of sellers asked if MCs had seen a doctor ( $12 \%$ of ADDOs and $19 \%$ of pharmacies in Mwanza, Tanzania and $25 \%$ of drug shops and $24 \%$ of pharmacies in Mbarara, Uganda). Critically, only about 3\% of ADDOs and 7\% of pharmacies in Mwanza, Tanzania asked the MC if they had a prescription compared to about $11 \%$ of drug shops and $14 \%$ of pharmacies in Mbarara, Uganda. In most cases, pharmaceutical drugs were sold; this was less common in pharmacies (60\%) compared to ADDOs (85\%) in Mwanza, Tanzania, but no such difference was observed between drug shops and pharmacies in Mbarara, Uganda (88\% vs $87 \%$ ). The majority of pharmaceutical drugs sold were prescription-only drugs.

Across both study sites, nearly $90 \%$ of drug sellers who sold prescription-only medicines agreed to the MCs request for 'only a few days' worth (recorded as 'sold a partial course'). The situation was less 
common in Uganda, where $42 \%$ of drug shops and $31 \%$ of pharmacies that sold prescription-only medicines sold a 'partial course' without further questions. In Tanzania, however, $67 \%$ of ADDOs and $51 \%$ of pharmacies that sold prescription-only medicines sold a 'partial course' without challenging the MC's request. In Uganda, $55 \%$ of drug shops and $56 \%$ of pharmacies that sold prescription-only medicines hesitated before dispensing a partial course, whereas in Tanzania only $32 \%$ of ADDOs and $49 \%$ of pharmacies did so. Drug sellers in Mbarara, Uganda were more likely to advise completing the course of prescription-only drugs sold (45\% in both seller types) than those in Mwanza, Tanzania (19\% of ADDOs and $14 \%$ of pharmacies). Analysis of the mean number of tablets sold (see supplementary material, Table S1) shows that all sellers, regardless of the country and type of outlet, were likely to sell less than optimal numbers of tablets, and that mean numbers of tablets sold were lower in Mwanza, Tanzania than in Mbarara, Uganda.

\section{Characteristics of the drugs sold}

The majority of prescription-only drugs were antibiotics (Figure 4); a full list of the drugs dispensed by drug sellers and their WHO AWaRE classification are available in Supplementary file S2. Of those sellers who sold antibiotics, nearly all sold drugs that were on the AWaRE 'Access' list, which are of the lowest concern in preventing the spread of AMR. Only one ADDO among all surveyed drug sellers in Mwanza, Tanzania sold an antibiotic on the WHO AWaRE 'Watch' list, which has a higher potential to lead to resistance, compared to about $2 \%$ of pharmacies and drug shops in Mbarara, Uganda. Nearly $80 \%$ of drug sellers in Mbarara, Uganda sold antibiotics that were compatible with national guidelines for treating secondary bacterial infections in COVID-19 patients, compared to less than half of the pharmacies (40\%) and ADDOs (46\%) in Mwanza, Tanzania. This distribution was, however, higher among drug sellers that suspected COVID-19. About $75 \%$ of pharmacies that sold antibiotics and suspected COVID-19 in Tanzania sold antibiotics listed as relevant for treating secondary bacterial infections in hospitalised patients with severe COVID-19.

\section{Discussion}

Our study sought to investigate drug dispensing practices in Mbarara, Uganda and Mwanza, Tanzania, during the COVID-19 pandemic and to consider the implications of these practices for AMR. Mystery clients presented with COVID-19-like symptoms in two LMIC settings with different policy responses to the COVID-19 pandemic. Our analysis uncovers four main findings. First, drug sellers' COVID-19 vigilance and prevention advice were more common in Uganda, which has experienced strict lockdowns and a more consistent policy response than in Tanzania. Nevertheless, given that MCs presented with potential COVID-19 symptoms in the midst of a pandemic, it is significant that awareness and vigilance among sellers were below $50 \%$ in both settings. Second, we observed poor compliance with antibiotic dispensing regulations in both sites and in all types of outlets. The majority of pharmacies and drug shops/ADDOs dispensed prescription-only antibiotics without a prescription and sold a partial course without hesitation. This is similar to practices observed at the same sites in our earlier mystery client studies conducted pre- 
COVID-19 [27], and in other settings [22]. As research has shown, inappropriate consumption of antibiotics or consumption of partial courses has potentially wide-ranging implications for AMR [6-8]. Third, whether sellers interpreted the symptoms described as COVID-19 or a more common respiratory ailment, most dispensed an antibiotic for symptoms that could have been viral infection, which also has implications for inappropriate antibiotic consumption and AMR. Fourth, whilst most sellers in Mbarara, Uganda, and a large minority of those in Mwanza, Tanzania, sold an antibiotic that was nationally approved for the treatment of secondary bacterial infections among patients suffering severe COVID-19 symptoms, MCs were undiagnosed and presented as suffering only mild symptoms. This suggests that even if the propensity to dispense antibiotics without prescription has not changed markedly, the pandemic may still increase inappropriate use of antibiotics, which may exacerbate AMR and therefore reduce the efficacy of drugs used for the treatment of bacterial infection, including secondary infection in COVID-19 and other upper respiratory tract infections.

It is clear from this study and others $[22,27,28]$, that drug sellers in Uganda and Tanzania, like those in many other LMIC settings [40-43], do not always adhere to regulations on antibiotic dispensing, even though many conformed to other expectations of licensing and regulation. Pharmacies, where sellers might be expected to have a higher degree of training and awareness of regulation, were only marginally better at following dispensing guidelines than drug shops or ADDOs. However, those who are licensed may not always be the ones to operate the pharmacies or drug shops, as highlighted in several studies $[44,45]$. This suggests that, in order to be effective, interventions should be aimed at all seller types, rather than targeting particular types of drug sellers.

Many existing studies suggest that the lack of legal consequences for inappropriate prescription and dispensing of antibiotics is a key factor for the dispensing practices we observed in this study [41,46-48], which leads researchers to emphasise the need for the enforcement of regulations, stricter surveillance of drug sellers' practices and more severe sanctions for poor practice. However, there is limited evidence that enforcement of regulations alone can successfully improve antibiotic stewardship outside of a suite of multifaceted measures aimed at all stakeholders, including sellers, upstream actors like pharmaceutical suppliers, and clients themselves [49]. A plethora of factors drive drug seller behaviours. Drug sellers' motivations to dispense include pressure from both patients and suppliers $[4,22,44,50]$, which emphasises the relationality of the encounter and the supply-demand dynamics at play. Pharmaceutical companies and their representatives are also likely to pressure, encourage or incentivise drug sellers to prescribe or dispense specific brands of antibiotics [48]. At the same time, drug sellers are also likely to have commercial interests in complying with their customers' requests, including both the need to profit [51] and building goodwill to ensure repeat visits in the future [44]. Thus, further work should address the interrelated contextual determinants of drug seller practices in LMICs to design more effective interventions. One example would be upskilling and knowledge training among drug sellers. For example, Kitutu et al. [52] report an intervention in Uganda, which successfully motivated adherence to recognised guidelines by engaging drug sellers as partners to enhance effective treatment for uncomplicated malaria. 
Lastly, our finding on the differential compliance with internationally recognised preventative measures for curtailing the spread of COVID-19 (such as handwashing and wearing a mask) reveals how national policy responses shaped individuals' behaviours and perceptions of risk. Other studies in Tanzania have observed limited social distancing and preventative measures [33]. This contrasts with Uganda, where more stringent policies and public health messaging were adopted, including school closures, household lockdowns and hygiene measures. The Tanzanian government emphasised the protection of citizens' livelihoods and therefore did not aim to fundamentally change their behaviour. This national response may have shaped population-level awareness of the virus.

This study is the first to investigate drug seller practices during the COVID-19 pandemic in Uganda and Tanzania using a mystery client approach. Despite its contributions, it is not without its limitations. First, although we used a comprehensive sample frame of sellers, ground-tested and used in other studies [27], we subsequently selected $75 \%$ of the original sample frame using convenience sampling, which could have led to sampling bias. Relatedly, although the study sample comprised one region in each country, and hence might not be generalisable, these regions did comprise both urban and rural areas, and our previous study suggests that patterns of response in the two sites are mirrored in the other country sites [27]. Second, the study used a 'mystery client' approach which relied on MCs' subjective assessment of their interactions with drug sellers. For example, MCs reported whether drug sellers seemed shocked or alarmed at the mention of COVID-19 symptoms. They may not have been able to adequately capture the sellers' reaction because some drug sellers, particularly in Tanzania, may have avoided mentioning COVID-19, even if they suspected it, to avoid potential repercussions for themselves and their business, particularly in settings characterised by high COVID-19 denial. Consequently, there may be a possibility that this study undercounted the sellers who suspected COVID-19. In addition, the scenario of presenting with respiratory symptoms may have been challenging to simulate realistically, leading sellers to suspect a milder ailment. Finally, MCs in Tanzania did not include the 'loss of taste/smell' in their symptom presentation to the drug sellers because, at the time, this was not locally recognised as a COVID-19 symptom. This may have affected the sellers' response to MCs, including the likelihood of suspecting COVID-19, the advice given and the drugs dispensed. Nonetheless, MCs presented with upper respiratory tract symptoms in the midst of a global viral respiratory pandemic; therefore, it would be reasonable to expect drug sellers to recognise the possibility of infection, which therefore upholds the validity of our results.

\section{Conclusion And Implications}

Drug sellers play a vital role in providing the public with access to antibiotics in LMIC settings. This study confirms that the patterns of antibiotic dispensing by drug sellers observed previously, which potentially place both patients and medicines at risk, have not altered during the COVID-19 pandemic. When presented with symptoms commensurate with COVID-19 / upper respiratory tract infection, with no indication of bacterial origin, drug sellers commonly sold antibiotics without a prescription. This suggests that, as COVID-19 spreads in communities and more people present with such symptoms, (inappropriate) antibiotic dispensing may become more frequent, which may further drive AMR, including in drugs vital 
for treating secondary infections in COVID-19 patients. Moreover, despite MCs presenting with COVID-19like symptoms, the sellers suspected COVID-19 in very few cases and did not recommend infection prevention measures, which may have implications for COVID-19 transmission.

Furthermore, many sellers dispensed prescription-only antibiotics approved for treating bacterial coinfection in patients more likely to manifest in patients experiencing severe COVID-19. Such prophylactic use is a misapplication in both clinical and regulatory terms and opens new avenues for the development of AMR to the drugs concerned. Overall, our findings suggest that existing systems designed to regulate antibiotic use, prevent AMR, and halt the spread of COVID-19 are not robust. To address this, recognising drug sellers' unique and indispensable role as first points of call in these communities may be equally as productive as restriction and sanction. Drug sellers in LMICs are uniquely positioned to act as an important first-line protection mechanism against both COVID-19 spread, and inappropriate antibiotic dispensing, and solutions may come in thinking about drug sellers' role in the healthcare system inclusively.

\section{Abbreviations}

CARE: COVID-19 and Antimicrobial Resistance in East Africa

HATUA: Holistic Approach to Unravel Antibacterial Resistance in East Africa

GPS: Global Positioning System

Pharm: Pharmacy

DS: Drug shop

ADDO: Accredited drug dispensing outlet

AMR: Antimicrobial Resistance

MC: Mystery client

\section{Declarations}

\section{Availability of data and materials}

The datasets supporting the conclusions of this article are available from the lead authors upon a reasonable request.

\section{Acknowledgements}


We would like to thank the CARE field teams in Mbarara, Uganda and Mwanza, Tanzania for conducting the survey during the COVID-19 pandemic.

\section{Funding}

CARE: COVID-19 and Antimicrobial Resistance in East Africa - impact and response is a Global Effort on COVID-19 (GECO) Health Research Award (MR/V036157/1) funded by UK Research and Innovation (Medical Research Council) and the Department of Health and Social Care (National Institute for Health Research).

\section{Authors' contribution}

EO co-led the data analysis, and wrote the manuscript. ETK co-led the data analysis and edited the manuscript. CK co-led the data analysis and edited the manuscript. MK co-designed the study, and edited the manuscript. KK co-designed the study, supervised data analysis, and edited the manuscript. SN codesigned the study, supervised data collection and reviewed the manuscript. BA co-designed the study and reviewed the manuscript. SEM co-designed the study and reviewed the manuscript. MFM codesigned the study, supervised data collection, and reviewed the manuscript. OL helped design the data collection tools and reviewed the manuscript. BS supervised data collection in Uganda and reviewed the manuscript. AS co-designed and coordinated the study, and reviewed the manuscript. DJS co-designed the study and reviewed the manuscript. FB-P developed maps of the sampling frame and reviewed the manuscript. JRM co-designed the study, supervised data collection and reviewed the manuscript. WS codesigned the study and reviewed the manuscript. MTGH led the design of the project, reviewed the manuscript, and acts as a guarantor of the work.

\section{Ethical statement}

The study received ethical approval from the University of St Andrews, UK (number MD15722, 31/08/21); National Institute for Medical Research, Tanzania (number 2831, updated 17/06/21); CUHAS/BMC Research Ethics and Review Committee (number CREC /266/2018, updated on 09/02/2021); Uganda National Council for Science and Technology (number HS1519ES, 22/07/21); and Makerere University, Uganda (number SBS-2021-31, 24/05/21).

\section{Consent for publication}

Not applicable.

\section{Competing interests}


The authors declare that they have no competing interests.

\section{References}

1 Dowd JB, Andriano L, Brazel DM, et al. Demographic science aids in understanding the spread and fatality rates of COVID-19. Proc Natl Acad Sci 2020;117:9696-8. doi:10.1073/pnas.2004911117

2 Wamai RG, Hirsch JL, Van Damme W, et al. What Could Explain the Lower COVID-19 Burden in Africa despite Considerable Circulation of the SARS-CoV-2 Virus? Int J Environ Res Public Health 2021;18:8638. doi:10.3390/ijerph18168638

3 Laxminarayan R, Van Boeckel T, Frost I, et al. The Lancet Infectious Diseases Commission on antimicrobial resistance: 6 years later. Lancet Infect Dis 2020;20:e51-60. doi:10.1016/S14733099(20)30003-7

4 Laxminarayan R, Duse A, Wattal C, et al. Antibiotic resistance-the need for global solutions. Lancet Infect Dis 2013;13:1057-98. doi:10.1016/S1473-3099(13)70318-9

5 Katale BZ, Misinzo G, Mshana SE, et al. Genetic diversity and risk factors for the transmission of antimicrobial resistance across human, animals and environmental compartments in East Africa: a review. Antimicrob Resist Infect Control 2020;9:127. doi:10.1186/s13756-020-00786-7

6 Auta A, Hadi MA, Oga E, et al. Global access to antibiotics without prescription in community pharmacies: A systematic review and meta-analysis. J Infect 2019;78:8-18.

doi:10.1016/j.jinf.2018.07.001

7 Saleem Z, Hassali MA, Hashmi FK, et al. Antimicrobial dispensing practices and determinants of antimicrobial resistance: a qualitative study among community pharmacists in Pakistan. Fam Med Community Heal 2019;7:e000138. doi:10.1136/fmch-2019-000138

8 Morgan DJ, Okeke IN, Laxminarayan R, et al. Non-prescription antimicrobial use worldwide: a systematic review. Lancet Infect Dis 2011;11:692-701. doi:10.1016/S1473-3099(11)70054-8

9 Monnet DL, Harbarth S. Will coronavirus disease (COVID-19) have an impact on antimicrobial resistance? Eurosurveillance 2020;25:1. doi:10.2807/1560-7917.ES.2020.25.45.2001886

10 Rafiq K, Nesar S, Anser H, et al. Self-Medication in the COVID-19 Pandemic: Survival of the Fittest. Disaster Med Public Health Prep 2021;:1-5. doi:10.1017/DMP.2021.173

11 Afolabi S, Folorunso SO, Bunyula ZS, et al. Social Listening: A Thematic Analysis of COVID-19 Discussion on Social Media. medRxiv 2020;:2020.07.25.20162040. doi:10.1101/2020.07.25.20162040

12 Arora A, Jha AK, Alat P, et al. Understanding coronaphobia. Asian J Psychiatr 2020;54:102384. doi:10.1016/J.AJP.2020.102384 
13 Rao SPN, Minckas N, Medvedev MM, et al. Small and sick newborn care during the COVID-19 pandemic: global survey and thematic analysis of healthcare providers' voices and experiences. $B M J$ Glob Heal 2021;6:e004347. doi:10.1136/BMJGH-2020-004347

14 Zarocostas J. How to fight an infodemic. Lancet 2020;395:676. doi:10.1016/S0140-6736(20)30461-X 15 Arshad M, Mahmood SF, Khan M, et al. Covid -19, misinformation, and antimicrobial resistance. BMJ 2020;:m4501. doi:10.1136/bmj.m4501

16 Butler CC, Dorward J, Yu L-M, et al. Azithromycin for community treatment of suspected COVID-19 in people at increased risk of an adverse clinical course in the UK (PRINCIPLE): a randomised, controlled, open-label, adaptive platform trial. Lancet 2021;397:1063-74. doi:10.1016/S0140-6736(21)00461-X

17 Erku DA, Belachew SA, Abrha S, et al. When fear and misinformation go viral: Pharmacists' role in deterring medication misinformation during the 'infodemic' surrounding COVID-19. Res Soc Adm Pharm 2021;17:1954-63. doi:10.1016/J.SAPHARM.2020.04.032

18 Okereke M, Ukor NA, Ngaruiya LM, et al. COVID-19 Misinformation and Infodemic in Rural Africa. Am J Trop Med Hyg 2021;104:453-6. doi:10.4269/AJTMH.20-1488

19 Rutta E, Senauer K, Johnson K, et al. Creating a New Class of Pharmaceutical Services Provider for Underserved Areas: The Tanzania Accredited Drug Dispensing Outlet Experience. Prog Community Heal Partnerships Res Educ Action 2009;3:145-53. doi:10.1353/cpr.0.0063

20 Embrey M, Mbwasi R, Shekalaghe E, et al. National Health Insurance Fund's relationship to retail drug outlets: a Tanzania case study. J Pharm Policy Pract 2021141 2021;14:1-12. doi:10.1186/S40545-02100303-0

21 Chalker JC, Vialle-Valentin C, Liana J, et al. What roles do accredited drug dispensing outlets in Tanzania play in facilitating access to antimicrobials? Results of a multi-method analysis. Antimicrob Resist Infect Control 201541 2015;4:1-11. doi:10.1186/S13756-015-0075-2

22 Mayora C, Kitutu FE, Kandala N-B, et al. Private retail drug shops: what they are, how they operate, and implications for health care delivery in rural Uganda. BMC Heal Serv Res 2018181 2018;18:1-12. doi:10.1186/S12913-018-3343-Z

23 Awor P, Wamani H, Bwire G, et al. Private Sector Drug Shops in Integrated Community Case Management of Malaria, Pneumonia, and Diarrhea in Children in Uganda. Am J Trop Med Hyg 2012;87:92-6. doi:10.4269/AJTMH.2012.11-0791

24 Konde-Lule J, Gitta SN, Lindfors A, et al. Private and public health care in rural areas of Uganda. BMC Int Heal Hum Rights 2010101 2010;10:1-8. doi:10.1186/1472-698X-10-29 
25 Bagonza A, Peterson S, Mårtensson A, et al. Regulatory inspection of registered private drug shops in East-Central Uganda - What it is versus what it should be: A qualitative study. J Pharm Policy Pract 2020;13:1-11. doi:10.1186/S40545-020-00265-9/METRICS

26 Mbonye AK, Buregyeya E, Rutebemberwa E, et al. Prescription for antibiotics at drug shops and strategies to improve quality of care and patient safety: a cross-sectional survey in the private sector in Uganda. BMJ Open 2016;6:e010632. doi:10.1136/BMJOPEN-2015-010632

27 Ndaki PM, Mushi MF, Mwanga JR, et al. Dispensing Antibiotics without Prescription at Community Pharmacies and Accredited Drug Dispensing Outlets in Tanzania: A Cross-Sectional Study. Antibiot 2021, Vol 10, Page 1025 2021;10:1025. doi:10.3390/ANTIBIOTICS10081025

28 Horumpende PG, Sonda TB, Zwetselaar M van, et al. Prescription and non-prescription antibiotic dispensing practices in part I and part II pharmacies in Moshi Municipality, Kilimanjaro Region in Tanzania: A simulated clients approach. PLoS One 2018;13:e0207465.

doi:10.1371/JOURNAL.PONE.0207465

29 Mbonye AK, Buregyeya E, Rutebemberwa E, et al. Prescription for antibiotics at drug shops and strategies to improve quality of care and patient safety: a cross-sectional survey in the private sector in Uganda. BMJ Open 2016;6:e010632. doi:10.1136/bmjopen-2015-010632

30 Ministry of Health. Uganda clinical guidelines. Kampala, Uganda: 2012.

31 Ndaki PM, Mushi MF, Mwanga JR, et al. Non-Prescription antibiotics dispensing practices in community and accredited drug dispensing outlets in Tanzania: A simulated Clients Approach. TBA 2022.

32 Asiimwe BB, Kiiru J, Mshana SE, et al. Protocol for an interdisciplinary cross-sectional study investigating the social, biological and community-level drivers of antimicrobial resistance (AMR): Holistic Approach to Unravel Antibacterial Resistance in East Africa (HATUA). BMJ Open 2021;11:e041418. doi:10.1136/BMJOPEN-2020-041418

33 Kuiper G. Emotional risk assessments in the field: Leaving Tanzania during the COVID-19 pandemic: https://doi.org/101177/14661381211002856 Published Online First: 17 March 2021. doi:10.1177/14661381211002856

34 Makoni M. Tanzania refuses COVID-19 vaccines. Lancet 2021;397:566. doi:10.1016/S01406736(21)00362-7

35 Buguzi S. Covid-19: Counting the cost of denial in Tanzania. BMJ 2021;373. doi:10.1136/BMJ.N1052

36 Islam N, Sharp SJ, Chowell G, et al. Physical distancing interventions and incidence of coronavirus disease 2019: natural experiment in 149 countries. BMJ 2020;370:2743. doi:10.1136/BMJ.M2743 
37 Aanensen DM, Huntley DM, Feil EJ, et al. EpiCollect: Linking Smartphones to Web Applications for Epidemiology, Ecology and Community Data Collection. PLoS One 2009;4:e6968. doi:10.1371/JOURNAL.PONE.0006968

38 National Drug Authority. Professional Guidelines 2018: Licensing, Renewal, and New Licenses for Class C Drug Shops. 2018.

39 World Health Organization. The 2019 WHO AWaRe classification of antibiotics for evaluation and monitoring of use. Geneva: 2019.

40 Auta A, Hadi MA, Oga E, et al. Global access to antibiotics without prescription in community pharmacies: A systematic review and meta-analysis. J Infect 2019;78:8-18.

doi:10.1016/J.JINF.2018.07.001

41 Gebretekle GB, Serbessa MK. Exploration of over the counter sales of antibiotics in community pharmacies of Addis Ababa, Ethiopia: Pharmacy professionals' perspective. Antimicrob Resist Infect Control 2016;5:1-7. doi:10.1186/S13756-016-0101-Z/METRICS

42 Do NTT, Vu HTL, Nguyen CTK, et al. Community-based antibiotic access and use in six low-income and middle-income countries: a mixed-method approach. Lancet Glob Heal 2021;9:e610-9. doi:10.1016/S2214-109X(21)00024-3

43 Afari-Asiedu S, Kinsman J, Boamah-Kaali E, et al. To sell or not to sell; The differences between regulatory and community demands regarding access to antibiotics in rural Ghana. $J$ Pharm Policy Pract 2018;11:1-10. doi:10.1186/S40545-018-0158-6/TABLES/1

44 Adhikari B, Pokharel S, Raut S, et al. Why do people purchase antibiotics over-the-counter? A qualitative study with patients, clinicians and dispensers in central, eastern and western Nepal. BMJ Glob Heal 2021;6:e005829. doi:10.1136/BMJGH-2021-005829

45 Minzi OMS, Haule AF. Poor knowledge on new malaria treatment guidelines among drug dispensers in private pharmacies in Tanzania: the need for involving the private sector in policy preparations and implementation. East Afr J Public Health 2008;5:117-

21.http://www.ncbi.nlm.nih.gov/pubmed/19024421

46 Reynolds L, McKee M. Factors influencing antibiotic prescribing in China: An exploratory analysis. Health Policy (New York) 2009;90:32-6. doi:10.1016/J.HEALTHPOL.2008.09.002

47 Ayukekbong JA, Ntemgwa M, Atabe AN. The threat of antimicrobial resistance in developing countries: causes and control strategies. Antimicrob Resist Infect Control 201761 2017;6:1-8. doi:10.1186/S13756-017-0208-X

48 Wilkinson A, Ebata A, Macgregor H. Interventions to Reduce Antibiotic Prescribing in LMICs: A Scoping Review of Evidence from Human and Animal Health Systems. Antibiot 2019, Vol 8, Page 22018;8:2. 
49 Jacobs TG, Robertson J, van den Ham HA, et al. Assessing the impact of law enforcement to reduce over-the-counter (OTC) sales of antibiotics in low- and middle-income countries; a systematic literature review. BMC Heal Serv Res 2019191 2019;19:1-15. doi:10.1186/S12913-019-4359-8

50 Radyowijati A, Haak H. Improving antibiotic use in low-income countries: an overview of evidence on determinants. Soc Sci Med 2003;57:733-44. doi:10.1016/S0277-9536(02)00422-7

51 Emgård M, Mwangi R, Mayo C, et al. Tanzanian primary healthcare workers' experiences of antibiotic prescription and understanding of antibiotic resistance in common childhood infections: a qualitative phenomenographic study. Antimicrob Resist Infect Control 2021101 2021;10:1-15. doi:10.1186/S13756021-00952-5

52 Kitutu FE, Kalyango JN, Mayora C, et al. Integrated community case management by drug sellers influences appropriate treatment of paediatric febrile illness in South Western Uganda: a quasiexperimental study. Malar J 2017161 2017;16:1-18. doi:10.1186/S12936-017-2072-9

\section{Figures}

\section{Figure 1}

Geographic distribution of drug sellers sampled in the survey 
Drug seller suspected COVID

Drug seller seemed shocked/alarmed

Drug seller advised mystery shopper to wear face mask

Drug seller advised mystery shopper to social distance

Drug seller advised mystery shopper to self isolate

Drug seller advised mystery shopper to get tested

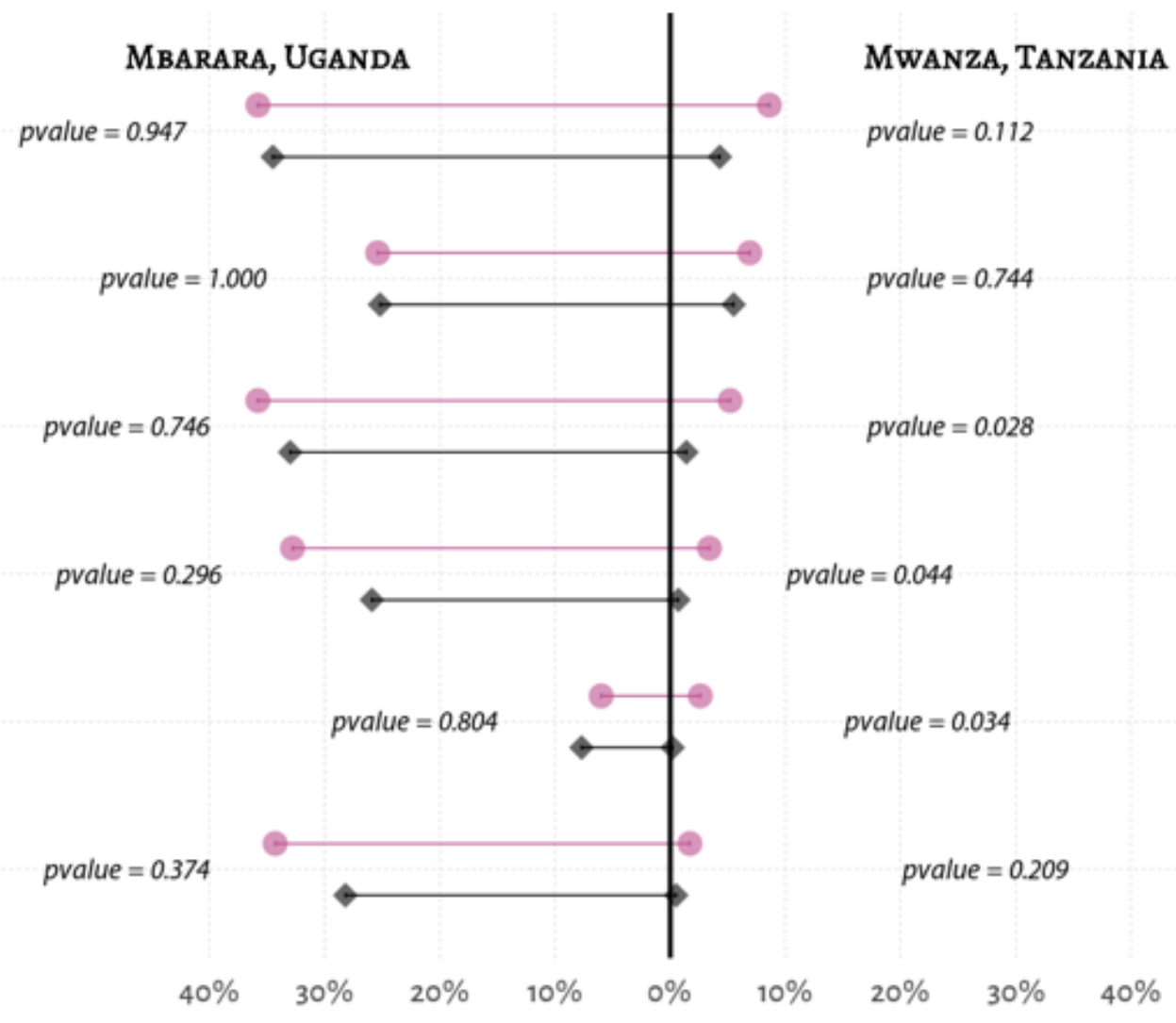

ADDO/Drug Shop
Pharmacy

\section{Figure 2}

Explicit and implicit vigilance to COVID-19 and recommendations for managing COVID-19-like symptoms between drug seller types in Mbarara, Uganda and Mwanza, Tanzania 

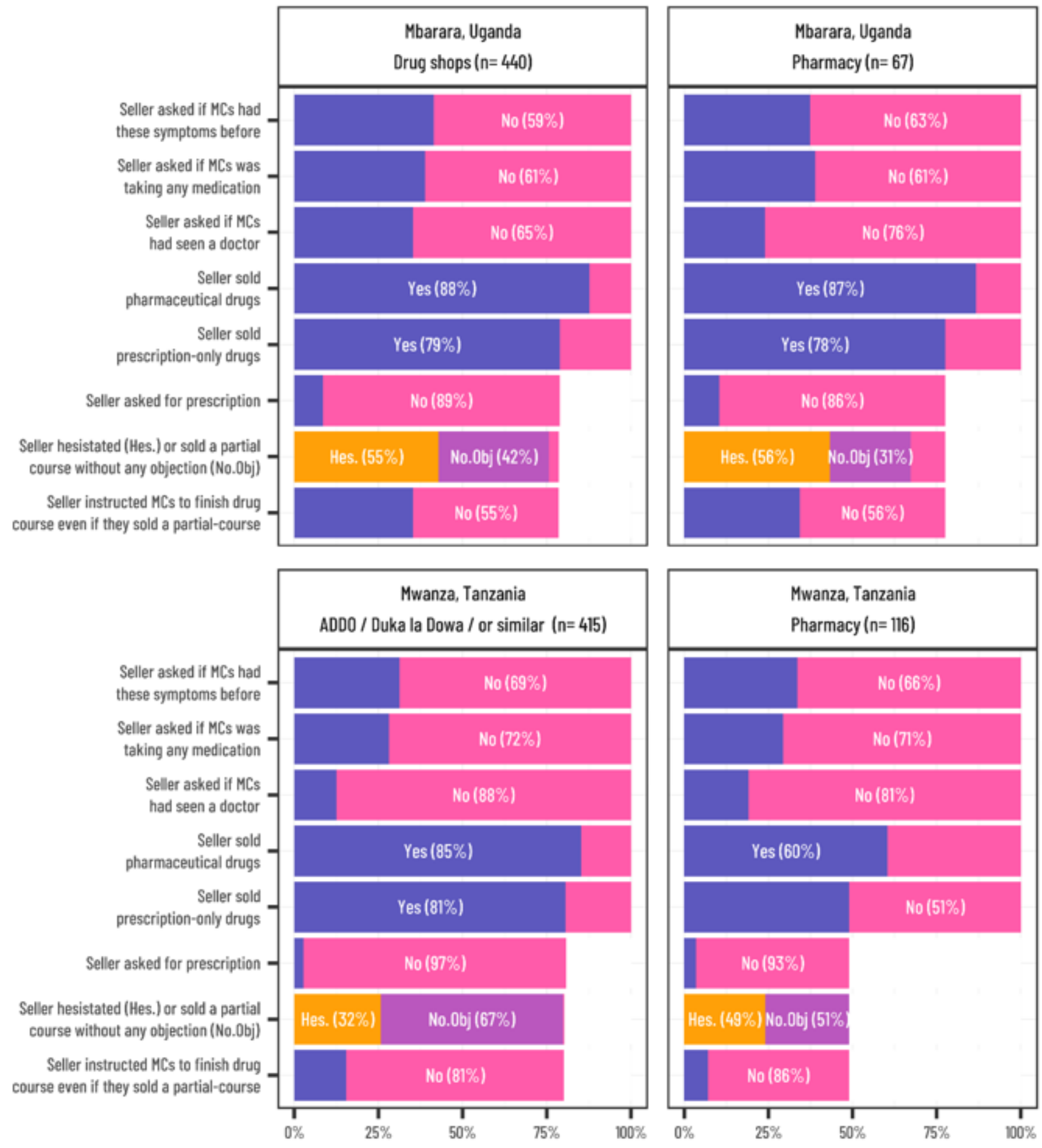

\section{Figure 3}

Patterns and quality of drug seller-client interaction by the quality of assessment, drug dispensing practices and advice. Note: No.Obj. = drug seller sold a partial course without any objection, Hes. = drug seller hesitated in selling half-course but still sold it. 


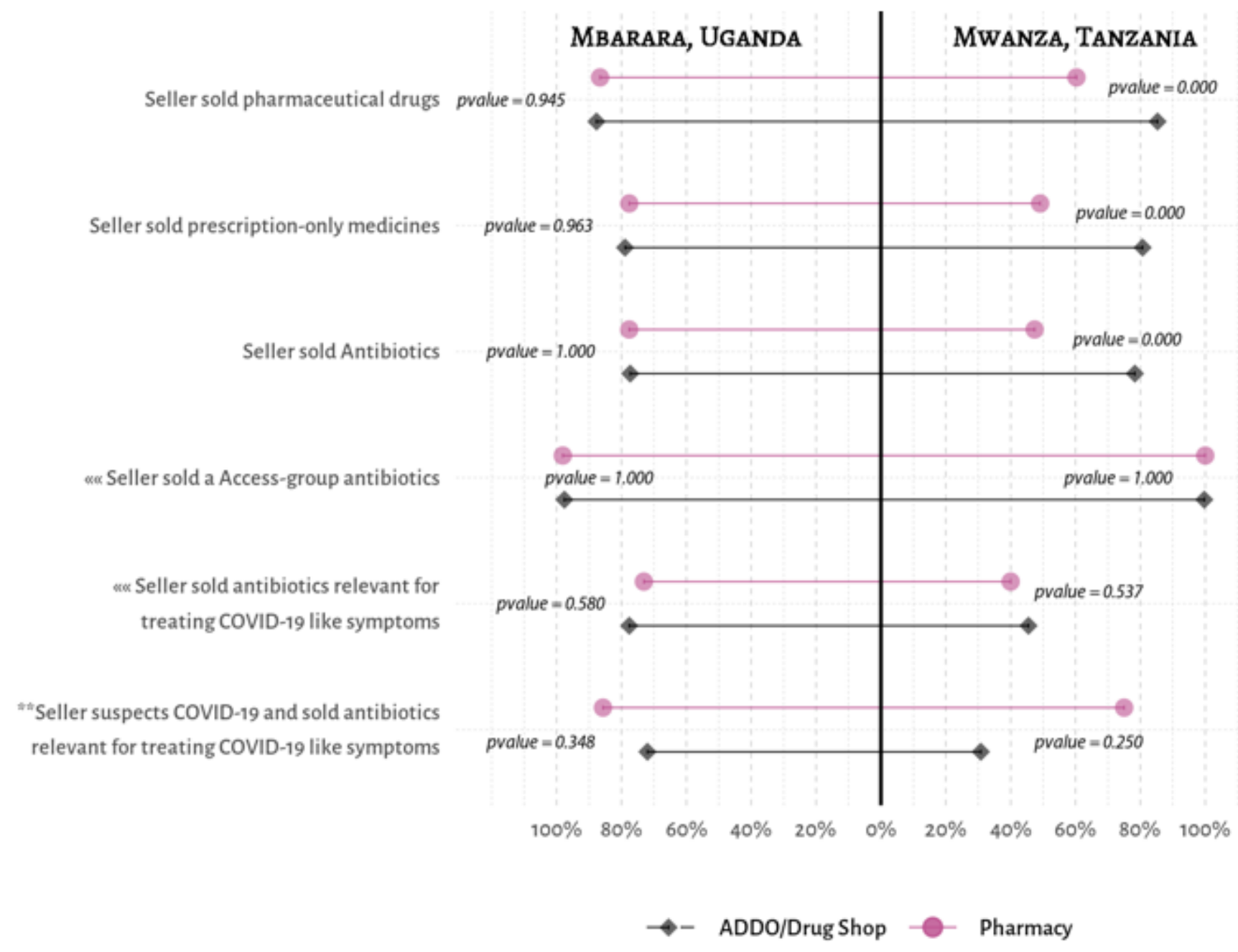

\section{Figure 4}

Comparison of drug-dispensing practices between pharmacies and drug shops/ADDOs in Tanzania and Uganda. Note: "« - implies that the percentage distribution is based on the total number of drug sellers who sold antibiotics; ** - implies that the percentage distribution is based on the total number of drug sellers who suspected COVID-19 and sold antibiotics

\section{Supplementary Files}

This is a list of supplementary files associated with this preprint. Click to download.

- Manuscriptsupplementaryfile.docx 